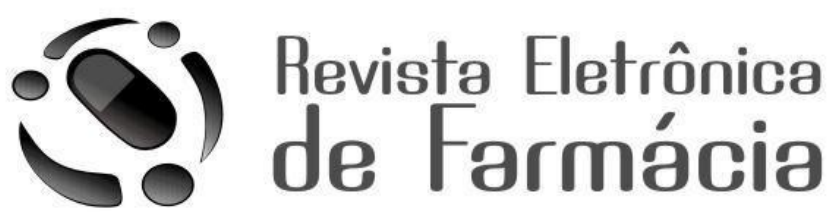

REF - ISSN 1808-0804 Vol. X (2), 24 - 41, 2013.

\title{
INVESTIGAÇÃO DE PROBLEMAS RELACIONADOS COM MEDICAMENTOS EM UMA INSTITUIÇÃO PA- RA LONGA PERMANÊNCIA PARA IDOSOS
}

INVESTIGATION OF RELATED PROBLEMS WITH DRUGS IN AN INSTITUTION FOR LONG STAY FOR ELDERLY

INVESTIGACIÓN DE LOS PROBLEMAS RELACIONADOS CON LAS DROGAS EN UNA INSTITUCIÓN DE LARGA ESTANCIA PARA PERSONAS MAYORES

\section{${ }^{1}$ Diêgo Martins de Oliveira Carvalho, ${ }^{1}$ Roberta Mayara de Moura Rocha, ${ }^{1 *}$ Rivelilson M Freitas}

${ }^{1}$ Curso de Farmácia da Universidade Federal do Piauí. Teresina, Piauí.

*autor para correspondência: rivelilson@pq.cnpq.br

Recebido em 24/11/2012, Aceito em 15/04/2013

\section{RESUMO}

Introdução. O aumento da longevidade e predomínio de doenças que exigem tratamento de longa duração, principalmente nos idosos, transforma-os no grupo etário mais medicalizado da sociedade. Assim, objetivou-se identificar patologias mais frequentes e classes farmacológicas mais prescritas entre idosos residentes na instituição de longa permanência, Associação Divina Providência (Casa São José), localizada no município de Teresina, Piauí, a avaliação das interações farmacológicas, detecção e classificação dos principais problemas relacionados com medicamentos (PRMs) encontrados. Métodos. Efetuou-se estudo exploratório descritivo em abordagem quantitativa, com análise 
CARVALHO, D.M.O.; ROCHA, R.M.M.; FREITAS, R.M. Revista Eletrônica de Farmácia Vol. X (2), $24-41,2013$.

prospectiva das fichas dos atendimentos aos idosos nos serviços de saúde e estudo dos prontuários. A população de referência foi de idosos da Casa São José. Resultados. Os PRMs mais encontrados foram medicamentos contra indicados $(36,84 \%)$ e interações medicamentosas $(29,82 \%)$, total de 57 casos. Destes, foi observado alto número de RNMs, total de 58 , dos quais $65,52 \%$ foram classificados quanto à segurança do medicamento ao paciente; $31,03 \%$ quanto à efetividade; e 3,45\% quanto à necessidade em tê-lo presente na farmacoterapia, igualmente distribuída entre os gêneros. Conclusões. Os principais PRMs detectados foram o uso de fármacos contra indicados para essa faixa etária e o número elevado de interações medicamentosas, resultado esperado pela presença de polifarmácia nos residentes estudados.

Descritores: Atenção farmacêutica, geriatria, reações adversas a medicamentos.

\section{ABSTRACT}

Introduction. Increased longevity and prevalence of diseases that require long-term treatment, especially the elderly, making them the age group most medicalized society. Aimed to identify the most frequent pathologies and the most prescribed classes of drugs among elderly residents in long-term institution, Divine Providence Association (Casa San Jose), located in the city of Teresina, Piauí, the evaluation of drug interactions detection and classification of major drug related problems (DRPs) found. Methods. Exploratory descriptive study was performed in a quantitative descriptive approach with prospective analysis of the forms of care for the elderly in health services and study of medical records. The reference population was elderly of St. Joseph House. Results. The DRPs were found more drugs indicated (36.84\%) and drug interactions (29.82\%), total of 57 cases. Of these, observed a high number of RNMs, 58 total, of which $65.52 \%$ were classified as to the safety of the drug to the patient; $31.03 \%$ for the effectiveness, and $3.45 \%$ on the need to have him present in pharmacotherapy, equally distributed between genders. Conclusions. The main DRPs identified were the use of drugs contraindicated for this age group and the high number of drug interactions, as expected by the presence of polypharmacy in residents studied.

Descriptors: Pharmaceutical care, geriatrics, adverse drug reactions.

\section{RESUMEN}

Introducción. Aumento de la longevidade y una prevalencia de las enfermedades que requieren tratamiento a largo plazo, especialmente los ancianos, por lo que el grupo de edad la mayoría de la sociedad medicalizada. Así, el objetivo fue identificar las patologías más frecuentes y las clases más prescritos de drogas entre los ancianos residentes en la institucion a largo plazo, la Divina Providencia de la Asociación (Casa San José), ubicado en la ciudad de Teresina, la evaluación de las interacciones entre medicamentos 
CARVALHO, D.M.O.; ROCHA, R.M.M.; FREITAS, R.M. Revista Eletrônica de Farmácia Vol. X (2), $24-41,2013$.

detección y clasificación de los principales problemas de las drogas relacionadas (PRM) encontrado. Métodos. Se realizó un estudio exploratorio descriptivo en un enfoque cuantitativo con el análisis prospectivo de las formas de atención a los ancianos en los servicios de salud y el estudio de las historias clínicas. La población de referencia fue los ancianos de San José. Resultados. Los PRM se encontraron más medicamentos indicados $(36,84 \%)$ y las interacciones medicamentosas $(29,82 \%)$, total de 57 casos. De éstos, observó un elevado número de RNMS, 58 total, de los cuales se clasificaron $65,52 \%$ en cuanto a la seguridad del fármaco al paciente; 31,03\% para la eficacia, y 3,45\% en la necesidad de tener lo presentan en tratamiento farmacológico, igualmente distribuidos entre géneros. Conclusiones. Los principales problemas relacionados con medicamentos identificados fueron el uso de medicamentos contraindicados para este grupo de edad y el elevado número de interacciones farmacológicas, como se esperaba por la presencia de la polifarmacia en los residentes estudiados.

Descriptores: La atención farmacéutica, geriatría, reacciones adversas a los medicamentos.

\section{INTRODUÇÃO}

O aumento da longevidade é um fenômeno mundial, e a faixa etária mais crescente no mundo é a de indivíduos com 80 anos ou mais ${ }^{(1)}$. A expectativa de vida e a qualidade da mesma aumentaram drasticamente nos últimos anos, e estes ganhos se devem a vários fatores, entre os quais a existência de milhares de medicamentos disponíveis para uso. Este uso da medicina conseguiu evitar causas de morte prematura, bem como controlar a doença e aliviar seus sintomas ${ }^{(2)}$.

Nesse processo observa-se a substituição, entre as primeiras causas de morte, de doenças transmissíveis por doenças não transmissíveis e por causas externas, além do deslocamento da maior carga de morbimortalidade dos segmentos mais jovens para os mais envelhecidos. Modifica-se, assim, o perfil de morbimortalidade da população, com predomínio de doenças que exigem tratamento de longa duração, e que frequentemente são acompanhadas de complicações secundárias ${ }^{(3)}$.

A doença crônica, via de regra, requer mudanças no estilo de vida, especialmente entre os idosos, além do acompanhamento da evolução do quadro clínico que, se não controlado adequadamente, tende a agravar o prognóstico. O aumento da prevalência das doenças crônicas não transmissíveis, na idade avançada, coloca os idosos no grupo etário mais medicalizado da sociedade ${ }^{(3)}$.

Fatores que acompanham o envelhecimento, como a maior prevalência de enfermidades crônicodegenerativas e consumo de fármacos, aumentam a incidência dos problemas 
CARVALHO, D.M.O.; ROCHA, R.M.M.; FREITAS, R.M. Revista Eletrônica de Farmácia Vol. X (2), $24-41,2013$.

relacionados aos medicamentos (PRM), bem como pode ser também potencializada pela diminuição do funcionamento de vários mecanismos fisiológicos, incluindo os relacionados à cinética e à dinâmica dos fármacos, deixando a população vulnerável aos vários problemas de saúde e aumentando os custos dos sistemas de atenção sanitária ${ }^{(4,5)}$.

A referida mudança gerou aumento na demanda por serviços e tecnologias de saúde, aumentando os custos assistenciais e exigindo dos gestores iniciativas custo-efetivas para minimizar os problemas decorrentes deste novo cenário sanitário(6).

Seguindo a tendência mundial, o Brasil movimenta uma intensa reestruturação na área do medicamento que permeia o Sistema Único de Saúde (SUS), envolvendo a formação e a prática dos profissionais de saúde, como também o bem estar e a qualidade de vida dos usuários do SUS. A implantação e implementação de ações preconizadas pelo SUS, a reestruturação das diretrizes curriculares, dos cursos da área de saúde, em especial a farmacêutica, a atuação conjunta da ANVISA, do Ministério da Saúde e da OPAS, vem fortalecendo as ações voltadas ao uso racional dos medicamentos, principalmente após a implantação da política de medicamentos genéricos ${ }^{(7)}$.

No Brasil, os medicamentos ocupam a primeira posição entre os causadores de intoxicações desde
$1996^{(8)}$. No entanto, há carência quase absoluta de estudos independentes na área de utilização de medicamentos no país, além da omissão do poder público no tratamento da problemática em questão ${ }^{(9)}$.

Vale ressaltar em relação à terapia medicamentosa, que antes de serem lançados no mercado, os medicamentos devem demonstrar que são seguros e eficazes, sendo necessária, para isso, a existência de uma regulamentação sanitária rígida. No entanto, todo este esforço para produzir medicamentos "teoricamente" seguros e efetivos, muitas vezes não se traduz em resultados positivos. Em vários casos, a farmacoterapia falha, ou seja, não atinge seus objetivos de aliviar um sintoma ou curar/controlar uma doença, podendo levar, inclusive, a efeitos indesejados. Justamente nestas situações, é importante que o farmacêutico desenvolva serviços como a Atenção Farmacêutica, sendo 0 seguimento farmacoterapêutico ideal para aqueles pacientes com doenças crônicas ${ }^{(10)}$.

Com o uso da polifarmácia, os idosos têm um risco maior de sofrer problemas relacionados a medicamentos (PRM), assim tendo uma baixa aderência ao tratamento. Os PRM's são classificados como um evento que pode ser identificado, prevenido e tratado e a avaliação de um PRM deve partir do princípio da indicação correta, verificando se o paciente sabe o motivo de estar utilizando o medicamento e 
CARVALHO, D.M.O.; ROCHA, R.M.M.; FREITAS, R.M. Revista Eletrônica de Farmácia Vol. X (2), $24-41,2013$.

também se o medicamento é eficaz e seguro. Com tudo, os PRM's têm como consequência o não alcance do objetivo do tratamento terapêutico e/ou o aparecimento de sintomas indesejáveis $^{(11)}$.

Através do acompanhamento farmacoterapêutico, o farmacêutico busca garantir o tratamento mais indicado, efetivo, seguro e conveniente a esses usuários, desta forma, a orientação do seu trabalho é deslocada do produto para o serviço e do medicamento para o paciente, considerando-o na sua totalidade, valorizando a ocorrência de problemas de saúde relacionados a medicamentos e buscando resolvê-los através da intervenção farmacêutica no contexto da equipe interdisciplinar, com a aplicação de medidas preventivas ou corretivas ${ }^{(12)}$.

Neste sentido, foi planejado desenvolver um estudo sobre a utilização de medicamentos em idosos, para identificar os principais PRM's, as patologias mais frequentes e as classes farmacológicas mais prescritas entre os idosos residentes na instituição da Associação Divina Providência (Casa São José), localizada no município de Teresina, Piauí.

\section{MÉTODOS}

Os dados foram coletados através da realização de um estudo do tipo exploratório descritivo em abordagem quantitativa, com emprego da técnica de observação direta através da análise prospectiva das fichas dos atendimentos realizados durante os atendimentos dos idosos nos serviços de saúde. Também foi realizado um estudo dos prontuários dos pacientes mediante a sua autorização, para coletar e registrar as informações referentes ao diagnóstico e a terapêuticas dos usuários em estudo. O projeto foi submetido e aprovado pelo Comitê de Ética em Pesquisa da Universidade Federal do Piauí (CEEA: 0308.0.045.000-10).

O trabalho foi realizado em uma instituição para longa permanência para idosos, Associação Divina Providência (Casa São José), no município de Teresina, durante o período de Agosto a Novembro de 2010. Essa instituição é filantrópica, sendo um local que presta assistência a 34 idosos. É composta por duas alas, masculina (17 idosos) e feminina (17 idosos). Os idosos recebem assistência médica de várias especialidades, como: clínica geral, psiquiatria, neurologia, oftalmologia, cardiologia, dentre outras. Além disso, eles recebem assistência material e espiritual, lazer e serviço funeral. A assistência médica é prestada por profissionais voluntários, que comparecem a casa semanalmente. A casa conta ainda com os serviços voluntários de uma fonoaudióloga e uma fisioterapeuta.

A amostra foi constituída por trinta e quatro (34) idosos atualmente cadastrados no serviço de cuidados da 
CARVALHO, D.M.O.; ROCHA, R.M.M.; FREITAS, R.M. Revista Eletrônica de Farmácia Vol. X (2), $24-41,2013$.

Casa São José em Teresina. Foram incluídos no estudo os idosos que fazem acompanhamento, residem na Casa São José e que aceitaram participar de forma voluntária. Não haverá exclusão em relação às características sócio demográficas dos idosos.

A coleta foi realizada durante o período de Setembro a Novembro de 2010, tendo por base os prontuários de cada um dos pacientes. A coleta dos dados dos idosos foi realizada por um acadêmico do Curso de Farmácia da Universidade Federal do Piauí, treinado e sob supervisão. Os registros dos dados foram feitos em formulários próprios específicos para o estudo por meio de entrevista com o idoso para se obter as seguintes variáveis: idade, principais problemas de saúde, hipóteses diagnósticas, interações medicamentosas, reações adversas a medicamentos, PRMs, medicamentos prescritos e os utilizados durante a prática da automedicação, além de analise dos prontuários de cada paciente estudado.

Além dos critérios de diagnóstico, ao analisarem-se os resultados, será importante considerar as seguintes variáveis:

a) Busca ativa dos casos - que será realizada através de entrevista direta e pessoal da amostra do estudo.

b) Composição da amostra - que obedecerá à estrutura da população quanto a sexo, idade, estrato socioeco- nômico, situação conjugal e inserção ocupacional.

c) Outras variáveis - quanto ao tipo de questionário empregado, a formação dos entrevistadores, a aceitação do estudo pela população e horário da coleta.

No tocante à entrevista farmacêutica como critério de exclusão foi considerado a incapacidade dos idosos em responder à ficha do seguimento farmacoterapêutico, aqueles que durante o período da coleta de dados não tivessem completado 60 anos, os que não se encontrassem efetivamente residindo na instituição ou os que evoluíram para óbito no período de realização do estudo. Portanto, no total, foram entrevistados 34 idosos, para iniciar o acompanhamento farmacoterapêutico. Em relação às análises de prescrições, foram analisadas as prescrições de todos os idosos.

\section{RESULTADOS e DISCUSSÃO}

Foram eleitos para o estudo indivíduos de ambos os sexos com idade igual ou superior a 60 anos residentes em uma instituição de longa permanência para idosos (ALPI) do município de Teresina, Piauí, composta por 34 residentes igualmente distribuídos entre alas masculina e feminina. Foram considerados como critérios de exclusão todos os eventos que comprometessem a permanência integral do residente na ALPI durante 0 
CARVALHO, D.M.O.; ROCHA, R.M.M.; FREITAS, R.M. Revista Eletrônica de Farmácia Vol. X (2), $24-41,2013$.

período de coleta de dados. Assim, oito $(23,52 \%)$ foram excluídos devido aos seguintes fatores: quatro por óbito, três por retornarem aos seus domicílios e um por idade inferior a 60 anos. Portanto, foram coletados e registrados os dados de um total de 26 residentes.

A distribuição dos idosos da amostra conforme parâmetros coletados relacionados à faixa etária e estado civil encontram-se demonstrado na Tabela 1. Há uma particularidade quanto a não predominância feminina na instituição estudada, visto que a divisão por alas com mesmo número de vagas para ambos os sexos, 17 homens e 17 mulheres, não permitem predominância feminina quando os residentes equivalem ao número máximo que a instituição comporta, revelando assim que os dados verificados não refletem o que foi documentado em outros trabalhos, em relação à predominância feminina em outras ALPI. Em um estudo sobre uso de medicamentos por idosos residentes em uma cidade do sul de Santa Catarina, as mulheres representavam $68,3 \%$ da população ${ }^{(13)}$. O estudo da faixa etária da população investigada revelou uma idade média de 73,3 anos (amplitude de 61 a 101 anos), com uma média de 73 anos para a população masculina e 81 anos para a feminina. Permitindo a observação de que as faixas etárias mais prevalentes compreendem o intervalo de 85 anos ou mais, e a distribuição da faixa etária no intervalo de 60 a 64 anos foi maior na ala masculina. Os dados encontrados neste estudo não corroboram com a literatura, uma vez que esta evidencia que quanto mais longínqua a população, maior a prevalência feminina, refletindo a estrutura social brasileira, em que mulheres atingem longevidade superior à do homem ${ }^{(14)}$.

Tabela 1 - Número absoluto e distribuição de frequência da população residente da ALPI conforme parâmetros de faixa etária e situação conjugal.

\begin{tabular}{|c|c|c|c|c|c|c|}
\hline \multirow[t]{2}{*}{ Parâmetros } & \multicolumn{2}{|c|}{$\begin{array}{l}\text { Masculino } \\
(n=12) \\
(46,15 \%)\end{array}$} & \multicolumn{2}{|c|}{$\begin{array}{l}\text { Feminino } \\
(n=14) \\
(53,85 \%)\end{array}$} & \multicolumn{2}{|c|}{$\begin{array}{c}\text { Total } \\
(n=26) \\
(100 \%)\end{array}$} \\
\hline & $\mathbf{N}$ & $\%$ & $\mathbf{N}$ & $\%$ & $\mathbf{N}$ & $\%$ \\
\hline \multicolumn{7}{|l|}{$\begin{array}{c}\text { FAIXA } \\
\text { ETÁRIA }\end{array}$} \\
\hline 60 a 64 anos & 5 & 19,23 & 0 & 0,00 & 5 & 19,23 \\
\hline 65 a 69 anos & 1 & 3,84 & 2 & 7,69 & 3 & 11,53 \\
\hline 70 a 74 anos & 2 & 7,69 & 2 & 7,69 & 4 & 15,38 \\
\hline 75 a 79 anos & 1 & 3,84 & 2 & 7,69 & 3 & 11,53 \\
\hline 80 a 84 anos & 1 & 3,84 & 3 & 11,53 & 4 & 15,38 \\
\hline
\end{tabular}


CARVALHO, D.M.O.; ROCHA, R.M.M.; FREITAS, R.M. Revista Eletrônica de Farmácia Vol. X (2), $24-41,2013$.

\begin{tabular}{ccccccr}
$\begin{array}{c}85 \text { anos ou mais } \\
\text { SITUAÇÃO } \\
\text { CoNJUGAL }\end{array}$ & 2 & 7,69 & 5 & 19,23 & 7 & 26,92 \\
Solteiros & 5 & 19,23 & 7 & 26,92 & 12 & 46,15 \\
\hline Casados & 3 & 11,53 & 1 & 3,84 & 4 & 15,38 \\
Viúvos & 1 & 3,84 & 5 & 19,23 & 6 & 23,07 \\
\hline Separados & 1 & 3,84 & 1 & 3,84 & 2 & 7,69 \\
Não informado & 2 & 7,69 & 0 & 0,00 & 2 & 7,69 \\
\hline
\end{tabular}

Considerando-se o estado civil, doze $(46,15 \%)$ da amostra era composta por solteiros, ao passo que seis $(23,07 \%)$ eram viúvos e quatro $(15,38 \%)$ casados. Dados em concordância com os estudos de SANTOS o qual afirma que as condições viúvos e solteiros, já eram esperadas em se tratando de um estudo realizado em ILP, onde muitos idosos foram encaminhados ou optaram por residir na instituição por apresentarem-se sem condições de residirem sós, seja por terem perdido o cônjuge ou por não terem filhos ${ }^{(15)}$.

Dos 26 idosos analisados durante 0 período estudado, pode-se observar que todos haviam recebido prescrição de algum tipo de medicamento alopático. Esse resultado confirma a noção de que a frequência de prescrições de medicamentos para cada idoso é consideravelmente elevada nesta faixa etária. Sabe-se que mesmo a prescrição aos idosos não institucionalizados costuma ser elevada. Um estudo nacional $^{(16)}$ com representação superior a 600 indivíduos verificou que $80,3 \%$, dos idosos utilizavam pelo menos um medicamento.
Neste trabalho, a amplitude do número de medicamentos prescritos por residente variou de 2 a 10 medicamentos entre os 26 idosos que receberam prescrições. Foi observado que para 9 idosos haviam sido prescritos de 1 a 4 medicamentos, enquanto 14 idosos haviam recebido prescrições de 5 a 8 medicamentos, e 3 haviam recebido nove ou dez mais prescrições simultaneamente (Figura 1).

Analisando-se as prescrições, observou-se um repertório de 142 diferentes especialidades farmacêuticas disponíveis no mercado nacional. Entre essas especialidades, foram contabilizados 79 medicamentos sob Nomenclatura Comercial e 63 foram prescritos pela Denominação Comum Brasileira. Em seu conjunto, esse repertório de medicamentos prescritos totalizou uma diversidade de 63 princípios ativos (PA) diferentes prescritos para a população estudada.

Foi observado que, do repertório de especialidades farmacêuticas prescritas, a maioria dos medicamentos, 92,95\% ( $\mathrm{n}$ = 132) era constituída por apenas um princípio ativo, enquanto $5,63 \%(n=8)$ 
CARVALHO, D.M.O.; ROCHA, R.M.M.; FREITAS, R.M. Revista Eletrônica de Farmácia Vol. X (2), $24-41,2013$.

possuíam dois e $1,40 \% \quad(n=2)$ possuíam 3 princípios ativos. Frequências semelhantes foram obtidas em um estudo realizado com uma população idosa residente em uma ALPI de Brasília, Distrito Federal, onde se verificou que $89,1 \%$ das especialidades farmacêuticas eram monodrogas ${ }^{(17)}$. Um fator que influencia nessa característica é que muitos idosos fazem uso de um um paciente tenha que fazer uso de uma associação de enalapril + hidroclorotiazida, por exemplo, é preferível que administre os fármacos separadamente, uma vez que além das monodrogas com menor custo, existem outros residentes que utilizam apenas um ou outro, dessa forma, há uma maior possibilidade de evitar o desperdício dos medicamentos.

mesmo medicamento, então mesmo que

$\square$ Número de Idosos

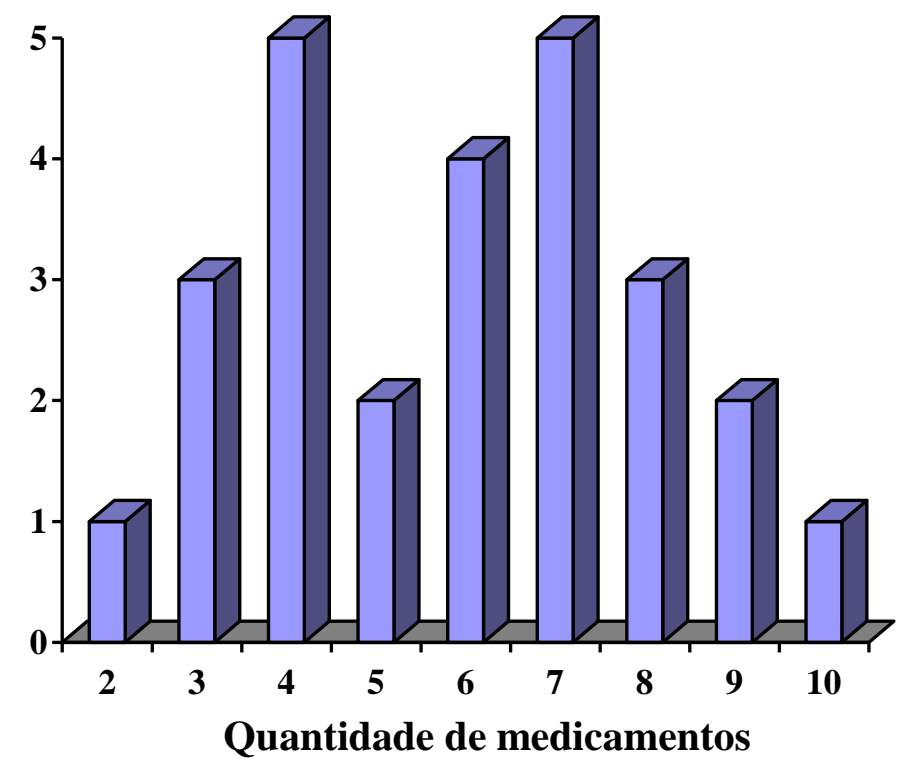

Figura 1 - Quantidade de medicamentos prescritos entre os idosos na ALPI.

Conforme descrito, o repertório de especialidades farmacêuticas prescritas aos residentes integralizava 63 princípios ativos diferentes. Considerando cada princípio ativo como um evento independente de medicação, foi observado um total de 154 eventos de medicação realizados para o grupo, o que determinou um número médio de 5,9 princípios ativos sendo prescrito a cada idoso residente da ALPI (Figura 2).
Observou-se uma influência do gênero, uma vez que o número de prescrições às mulheres foi superior ao número de prescrições aos idosos do sexo masculino. Esses achados reforçam os dados já existentes na literatura científica, corroborando para demonstrar que a prevalência de uso de medicamentos é maior no gênero feminino $^{(18)}$. 
CARVALHO, D.M.O.; ROCHA, R.M.M.; FREITAS, R.M. Revista Eletrônica de Farmácia Vol. X (2), $24-41,2013$.

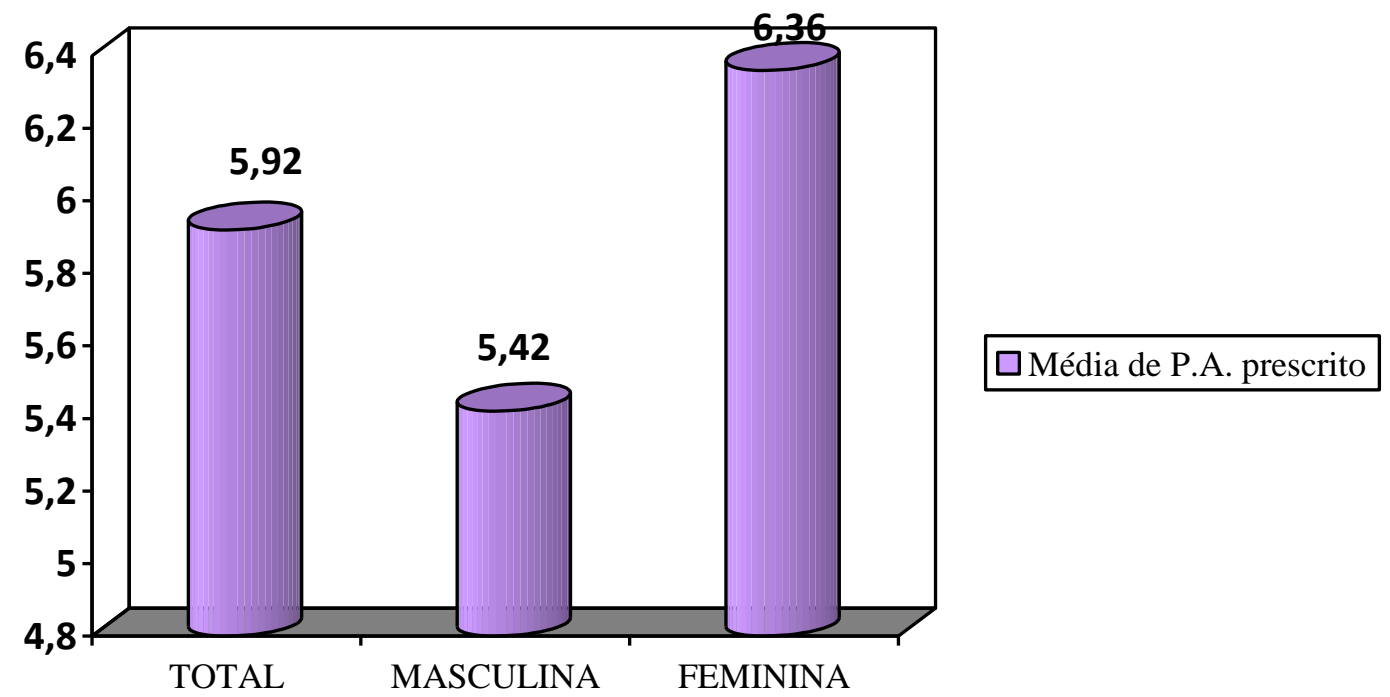

Figura 2 - Média total e por gênero de princípio ativo (P.A.) prescrito por/para os idosos residentes da ALPI.

Para esse estudo foi adotado como polifarmácia os casos em que os idosos residentes receberam simultaneamente mais de uma prescrição de princípios ativos diferentes. Fato observado em $100 \%$ da população estudada.

A análise dos princípios ativos mais prescritos revelou a prevalência do grupo de medicamentos com ação sobre o Sistema Nervoso Central (SNC) em que o diazepam (anticonvulsivante e ansiolítico) e fluoxetina (antidepressivo) foram os princípios ativos mais prescritos, correspondendo a $n=5$ e $n=$ 4, respectivamente. A prescrição de princípios ativos do grupo de medicamentos cardiovasculares também se mostrou elevada na população estudada, mas com menor número de variação de medicamentos, tendo como principais prescritos o inibidor da ECA enalapril ( $\mathrm{n}=6)$, o diurético hidroclorotiazida $(n=5)$ e o também inibidor da ECA captopril $(n=4)$. De acordo com a frequência dos princípios ativos prescritos, pode-se justificar o fato de os grupos farmacológicos mais prescritos correspondem ao grupo das drogas de ação sobre o SNC e os medicamentos cardiovasculares, responsáveis por $29,22 \%$ e $25,32 \%$ respectivamente, do total das prescrições feita a população em estudo (Tabela 2).

Tabela 2 - Número absoluto e distribuição de frequência dos eventos de prescrições dos grupos farmacológicos mais prevalentes entre os residentes da ALPI.

\begin{tabular}{ccc} 
Principais grupos farmacológicos & N & \% \\
\hline Drogas de ação sobre o Sistema Nervoso Central & 45 & 29,22 \\
Antihipertensivos, antianginosos, antiarrítimicos e diuréticos & 39 & 25,32
\end{tabular}


CARVALHO, D.M.O.; ROCHA, R.M.M.; FREITAS, R.M. Revista Eletrônica de Farmácia Vol. X (2), $24-41,2013$.

Antianêmicos, antihemorrágicos e antitrombóticos

$22 \quad 14,28$

Medicamentos de ação sobre o sistema músculo-esquelético

$08 \quad 5,19$

Analgésicos, antipiréticos e antiinflamatórios

$07 \quad 4,54$

Medicamentos que atuam sobre o trato digestório

$05 \quad 3,25$

Outros

$28 \quad 18,18$

Total

154100

Além das avaliações quanto às prescrições medicamentosas, foram analisados os prontuários dos residentes estudados para se obter o histórico de patologias, a frequência de administração de cada medicamento, duração e eventuais ocorrências registradas, como não adesão ao tratamento, forma de administração diferente da prescrita e comportamento do idoso ao decorrer do dia. Estas e outras observações cotidianas permitiram montar 0 quadro de problemas relacionados aos medicamentos com base na lista dos PRMs caracterizados no fórum de Atenção farmacêutica (2006), presentes no período do estudo realizado (Tabela $3)$.

Em outro estudo salientou-se que o uso simultâneo de medicamentos utilizados para diversos fins sugere 0 aparecimento de problemas relacionados à farmacoterapia. E propuseram que, além da polimedicação maior, o número de doses diárias, o uso de medicamentos de baixo índice terapêutico, o uso de medicamentos para vários problemas de saúde e a mudança na medicação são fatores de risco para o desenvolvimento de PRMs ${ }^{(13)}$.

De todos PRMs detectados, todos poderiam ser evitados ou ainda evitar um futuro resultado negativo associado aos medicamentos (RNM), uma vez que nenhum é associado às características pessoais do paciente, a conservação inadequada, bem com aos outros problemas de saúde que impossibilite o uso da farmacoterapia atual.

A administração dos medicamentos aos idosos é realizada por cuidadores ou técnicos de enfermagem, no entanto alguns pacientes apresentam certa resistência em realizar a farmacoterapia.

Tabela 3 - Problemas Relacionados aos Medicamentos (PRM) por gêneros encontrados no ALPI durante período de estudo realizado.

\begin{tabular}{|cccc|}
\hline $\begin{array}{c}\text { Problemas Relacionados aos } \\
\text { Medicamentos (PRMs) }\end{array}$ & Masculino & Feminino & N \\
\hline Administração errônea de medicamento & 01 & 02 & 03 \\
\hline
\end{tabular}


CARVALHO, D.M.O.; ROCHA, R.M.M.; FREITAS, R.M. Revista Eletrônica de Farmácia Vol. X (2), $24-41,2013$.

\begin{tabular}{|cccc|}
\hline Contra indicação & 09 & 12 & 21 \\
\hline Dose, pauta e/ou duração inadequada & 02 & 00 & 02 \\
\hline Duplicidade & 01 & 01 & 02 \\
\hline Erros de prescrição & 01 & 02 & 03 \\
\hline Não adesão & 05 & 04 & 09 \\
\hline Interações medicamentosas & 10 & 07 & 17 \\
\hline Total & $\mathbf{2 9}$ & $\mathbf{2 8}$ & $\mathbf{5 7}$ \\
\hline
\end{tabular}

Com orientação correta ao paciente idoso, este se faz entender o porquê do uso do medicamento, como vai atuar e que patologia vai tratar, por isso a importância do paciente ter todas as informações possíveis sobre sua patologia, seja ela temporária ou crônica, assim haverá uma relação de maior confiança com o profissional da saúde.

Dos PRMs encontrados, o farmacêutico ou qualquer outro profissional de saúde treinado poderia ter reduzido $24,56 \%$ dos PRMs listados ( $n=14$ ), apenas educando o paciente e o cuidador quanto ao tratamento realizado, aumentando o compromisso do idoso quanto à sua saúde. Desta maneira evitaria os PRMs causados por administrações errôneas; dose, pauta e/ou duração inadequada e aumentando a adesão do paciente à farmacoterapia.

Os quatro PRMs restantes encontrados estão relacionados à efetividade e segurança e requerem um pouco mais de informação e conhecimento a cerca dos princípios ativos (absorção, distribuição, finalidade e interações com outros medicamentos e/ou alimentos) por parte dos profissionais da saúde, principalmente médicos e farmacêuticos.

Os dois casos de duplicidade encontrados foram devidos a prescrições de médicos que não atuam presencialmente na instituição, assim, estes profissionais não tem acesso ao prontuário de cada paciente, e se este não for levado, haverá uma duplicidade em relação ao tratamento, já que são grande as chances de prescrição de um mesmo medicamento (por nome comercial diferente) ou ainda que pertençam a mesma classe, atuando de forma semelhante e potencializando o efeito esperado.

Os erros de prescrições ocorrem mais comumente quando se trata de um novo fármaco prescrito, pois nem médicos e farmacêuticos tem as informações consolidadas de todas as propriedades medicamentosas, como o melhor horário para administração, se há interação medicamentosa, entre outros.

Em um dos casos coletados, o fármaco digitálico digoxina estava sendo administrado juntamente ou logo após a refeição, no entanto a literatura revela que para uma melhor resposta terapêutica, este medicamento deve ser 
CARVALHO, D.M.O.; ROCHA, R.M.M.; FREITAS, R.M. Revista Eletrônica de Farmácia Vol. X (2), $24-41,2013$.

ingerido uma hora antes ou duas horas após refeição.

Publicações regulares de periódicos nacionais e internacionais, livros citados na bibliografia e o programa Micromedex foram utilizados como fonte de informação técnica, para detecção e avaliação das interações medicamentosas. Foram analisados os dados coletados referentes à farmacoterapia prescrita à população estudada, e assim, feita a classificação das interações encontradas em quatro tipos: contra-indicado, grave, moderada e branda (Tabela 4).

Desses, onze residentes $(40,74 \%)$ utilizavam uma combinação de P.A.s que apresentavam algum tipo de interação medicamentosa, quatro $(36,36 \%)$ pertencentes ao gênero masculino e sete $(63,64 \%)$ ao feminino. Fato este justificado pela maior média de princípios ativos serem consumidos entre as mulheres, portanto, ficando mais suscetíveis a uma eventual interação medicamentosa.

Tabela 4 - Classificação das interações medicamentosas detectadas a partir das prescrições da população residente da ALPI.

\begin{tabular}{|ccc|}
\hline Classificação das interações medicamentosas & $\mathbf{N}$ & $\mathbf{\%}$ \\
\hline Contra indicado & 00 & 0,00 \\
\hline Grave & 04 & 23,53 \\
\hline Moderada & 13 & 76,47 \\
\hline Branda & 00 & 0,00 \\
\hline Total & $\mathbf{1 7}$ & $\mathbf{1 0 0}$ \\
\hline
\end{tabular}

Os resultados dispostos configuram a ocorrência de polifarmácia iatrogênica, pela possibilidade de dois ou mais P.A.S distintos oferecidos ao mesmo paciente poderem expô-lo a efeitos adversos potencialmente perigosos causadores de comorbidades.

Pode-se deduzir que um maior número de P.A.s envolvidos em eventos de polifarmácia aumenta o risco de interações potencialmente perigosas, o que predispõe a população geriátrica à comorbidades associadas à medicação. Apesar de se compreender que esses achados não dependem necessariamente da quantidade de medicamentos administrados ao mesmo paciente, uma vez que guarda maior relação com a qualidade da prescrição realizada.

Avançando ainda mais sobre a investigação do valor terapêutico dos fármacos prescritos, foi analisada a frequência de prescrição de todos os medicamentos para avaliar o risco de efeitos adversos que excedam seus benefícios à saúde. De acordo com critérios de Fick $^{(19)}$ foi prescrito pelo menos um medicamento da categoria imprópria para 65,38\% $(n=17)$ da população estudada. Entre os que 
CARVALHO, D.M.O.; ROCHA, R.M.M.; FREITAS, R.M. Revista Eletrônica de Farmácia Vol. X (2), $24-41,2013$.

receberam essa categoria de medicação $76,47 \%$ ( $n=13)$ receberam prescrição de um, e 23,53\% ( $n=4)$ receberam prescrição de dois medicamentos impróprios para a faixa etária (Tabela 5).

Esses critérios abrangem apenas prescrições inapropriadas, não citando situações como fitoterápicos e subdoses medicamentosas. Percebe-se ainda, que os critérios de Fick não apontam para todas as situações que envolvem uso inapropriado de medicamentos em idosos no Brasil ${ }^{(19)}$.

Tabela 5 - Distribuição por gênero dos princípios ativos impróprios para idosos aos residentes da ALPI.

\begin{tabular}{|cccc|}
\hline $\begin{array}{c}\text { Princípios } \\
\text { ativos }\end{array}$ & Masculino & Feminino & Total \\
\hline Diazepan & 05 & 00 & 05 \\
\hline Fluoxetina & 00 & 04 & 04 \\
\hline Nimodipina & 01 & 02 & 03 \\
\hline Digoxina & 01 & 01 & 02 \\
\hline Orfenadrina & 00 & 02 & 02 \\
\hline Flurazepam & 00 & 01 & 01 \\
\hline Nifedipino & 00 & 01 & 01 \\
\hline Amiodarona & 01 & 00 & 01 \\
\hline Doxazosina & 01 & 00 & 01 \\
\hline Sulfato & 00 & 01 & 01 \\
\hline ferroso & & & $\mathbf{2 1}$ \\
\hline Total & $\mathbf{0 9}$ & $\mathbf{1 2}$ & \\
\hline
\end{tabular}

A partir das análises e distinção dos PRMs, pode-se relacionar os RNMs presentes ou que irão se manifestar com a continuidade da farmacoterapia atual. Ao todo, foram relacionados 58 RNMs, curiosamente presente entre os gêneros de forma igualitária, 29 por ala.

De acordo com 0 quadro 2 (Classificação dos Resultados Negativos associado ao Medicamento) foram listados e agrupados os RNMs do referido estudo, identificando-os quanto à necessidade, efetividade e segurança, e determinando quando trata-se de um evento quantitativo ou não quantitativo .

Dos 58 RNMs analisados, 65,52\% $(n=38)$ foram classificados quanto à segurança que o medicamento traz ao paciente; $31,03 \%(n=18)$ quanto à efetividade; e 3,45\% ( $n=2)$ quanto à necessidade em tê-lo presente em sua farmacoterapia.

A diferença de apresentação numérica entre PRM e RNM se justifica pelo fato de estes não possuírem uma relação de proporcionalidade "um pra 
CARVALHO, D.M.O.; ROCHA, R.M.M.; FREITAS, R.M. Revista Eletrônica de Farmácia Vol. X (2), $24-41,2013$.

um", ou seja, um PRM pode ocasionar um ou mais RNMs. Caso este coletado com a ocorrência de interação medicamentosa entre digoxina e maleato de timolol, que pode ocasionar bloqueio atrioventricular (insegurança nãoquantitativa) e aumento da toxicidade da digoxina (insegurança quantitativa), portanto, dois RNMs.

O número elevado relacionado à segurança está diretamente ligado à quantidade elevada de princípios ativos contra indicados, de acordo com BeersFick, que foram prescritos aos residentes. Nota-se que a falta de atualização quanto às propriedades farmacoterapêuticas que induz aos erros rotineiros, uma vez que muitos ainda tratam pacientes idosos como pacientes adultos comuns, não levando em consideração a fisiologia particular deste grupo.

Estes dados são suficientemente importantes para demonstrar a importância de um profissional farmacêutico atuando de forma mais próxima ao paciente, como em atenção domiciliar ou pela oferta de serviço de atenção farmacêutica em uma farmácia comunitária, seja ela pública ou privada, prevenindo comorbidades por iatrogenia.

O trabalho ainda demonstra a importância da caracterização de problemas relacionados aos medicamentos como medidas em saúde pública, uma vez que PRMs e RNMs estão entre as principais causas de internações e gastos hospitalares, inflando as filas de atendimento do Sistema Único de Saúde, que já é sobrecarregado.

\section{CONCLUSÃO}

Segundo o estudo, o perfil dos idosos atendidos pelos serviços de saúde da instituição de longa permanência Casa São José são de ambos os gêneros, divididos por alas de forma igualitária, a grande maioria acima de 60 anos e com baixa renda familiar. Os principais PRMs detectados foram o uso de fármacos contra indicados para essa faixa etária e o número elevado de interações medicamentosas, resultado esperado pela presença de polifarmácia em todos os residentes estudados. Destes, foram categorizados os RNMs presentes ou que ainda podem se manifestar, principalmente em relação à segurança dos medicamentos.

Ao se constatar a frequência de acontecimentos de problemas potenciais relacionados a fármacos (duplicidade farmacológica, prescrição de medicamentos contra indicados à faixa etária e interações medicamentosas potencialmente perigosas), pode-se concluir que as prescrições realizadas para essa população possuem valor terapêutico questionável quanto a determinados aspectos da medicação prescrita. Apesar de frequentes entre idosos, esses cenários provavelmente são agravados em instituições de longa permanência devido à inexistência de 
CARVALHO, D.M.O.; ROCHA, R.M.M.; FREITAS, R.M. Revista Eletrônica de Farmácia Vol. X (2), $24-41,2013$.

exigência legal por um quadro permanente de profissionais de saúde nestas instituições.

Esses dados fornecerão subsídios para a elaboração e implantação de intervenções farmacêuticas aos usuários idosos deste e de outras instituições de longa permanência.

\section{Conflitos de interesse: os} autores não possuem nenhum tipo de conflito de interesse a declarar em relação a este estudo.

\section{REFERÊNCIAS}

1. Nogueira SL, Ribeiro RCL, Rosado LEFP, Franceschini LSCC, Ribeiro AQ, Pereira ET. Fatores determinantes da capacidade funcional em idosos longevos. Rev. bras. Fisioter. 2010;14(4): 322-329.

2. Tercer Consenso de Granada sobre Problemas Relacionados con Medicamentos (PRM) y Resultados Negativos Asociados a la Medicación (RNM). Ars Phar. 2007;48 (1):5-17.

3. Cintra FA, Guariento ME, Miyasaki LA. Adesão medicamentosa em idosos em seguimento ambulatorial. Ciênc. Saúde Colet. 2010;15 (Supl. 3): 3507-3515.

4. Beserra A, Pompei LF, Camuzi RC. Atenção farmacêutica a pacientes idosos do hospital escola São Francisco de Assis, da Universidade Federal do Rio de Janeiro (UFRJ). In: 19a Semana Racine de Atualização em Farmácia; 2010; São Paulo.

5. Organização Pan-Americana da Saúde (org). Consenso Brasileiro de Atenção Farmacêutica: Proposta. Brasília: OPAS, 2002.

6. Carvalho FD, Artuzo FSC, Chrysostomo TN, Andrade RC. Influência do seguimento farmacoterapêutico sobre o tratamento medicamentoso de diabetes mellitus tipo 2 no brasil: revisão sistemática. Revista Brasileira de Farmácia Hospitalar e Serviços de Saúde. 2011;2 (2): 5-10.

7. BRASIL. Ministério da Saúde. Central de Medicamentos. $1^{\circ}$ Encontro Nacional de Assistência Farmacêutica e Política de Medicamentos. Brasília: Editora do Ministério da Saúde, 2001. 
CARVALHO, D.M.O.; ROCHA, R.M.M.; FREITAS, R.M. Revista Eletrônica de Farmácia Vol. X (2), $24-41,2013$.

8. Sistema Nacional de Informações Tóxico-Farmacológicas (SINITOX). Casos registrados de intoxicação e/ou envenenamento: Brasil, Rio de Janeiro: Ministério da Saúde / Fundação Oswaldo Cruz / Centro de Informações Científicas e Tecnológicas, 2008.

9. Lyra Junior DP, Amaral RT, Abriata JP, Pelá IR. A satisfação com resultado de um programa de atenção farmacêutica para pacientes idosos, em Ribeirão Preto (SP) Brasil. Pharmacy Practice. 2005;3 (1): 30-42.

10. Sousa IF, Bastos PRHO, Monreal MTFD, Hildebrand CR. Identificação de potenciais problemas relacionados com medicamentos: uma experiência de integração ensinoserviço na formação farmacêutica. Revista Eletrônica de Farmácia. 2012;IX(1): 40-48.

11. Couto CA, Christoff AO. Acompanhamento farmacoterapêutico em idosos de uma casa de longa permanência em curitiba-PR. Cadernos da Escola de Saúde. 2012;8:28-43.

12. Aires CCNF, Marchiorato LR. Acompanhamento farmacoterapêutico a hipertensos e diabéticos na unidade de saúde Tereza Barbosa: análise de caso. Revista Brasileira de Farmácia Hospitalar e Serviços de Saúde. 2010;1(1): 1-24.

13. Galato D, Silva ES, Tiburcio LS. Estudo de utilização de medicamentos em idosos residentes em uma cidade do sul de Santa Catarina (Brasil): um olhar sobre a polimedicação. Ciência \& Saúde Coletiva. 2010;15(6):2899-905.

14. Davim RMB, Torres GV, Dantas SMM, Lima VM. Estudo com idosos de instituições asilares no município de Natal/RN: características socioeconômicas e de saúde. Rev. Latino-Am. Enfermagem. 2004;12(3): 518-524.

15. Santos SSC, Feliciani AM, Silva BT. Perfil de idosos residentes em instituição de longa permanência: Proposta de ações de enfermagem/saúde. Rev. RENE. 2007;8(3):2633.

16. Coelho Filho JM, Marcopito LF, Castelo A. Perfil de utilização de medicamentos por idosos em área urbana do Nordeste do Brasil. Rev. Saúde Públ. 2004;38(4): 557-564.

17. Juarezi C, Margô GOK, Luvy G, Vianna OTN. Estudo da farmacoterapia prescrita a idosos em instituição brasileira de longa permanência. Acta Med Port. 2007;20: 97-105. 
CARVALHO, D.M.O.; ROCHA, R.M.M.; FREITAS, R.M. Revista Eletrônica de Farmácia Vol. X (2), $24-41,2013$.

18. Chrischilles EA, Lemke JH, Wallace RB, Drube GA. Prevalence and characteristics of multiple analgesic drug use in an elderly study group. J Am Geriatr Soc. 2004;38: 979-984.

19. Fick DM, Cooper JW, Wade WE, Waller JL, Maclean JR, Beers MH. Updating the Beers criteria for potentially inappropriate medication use in older adults: results of a US consensus panel of experts. Arch Intern Med. 2003;63: 2716-2724. 